\title{
Periodically Modulated Thermal Convection
}

Rui Yang $\odot{ }^{1,2, \dagger}$ Kai Leong Chong $\odot{ }^{1, \dagger}$ Qi Wang $\odot,{ }^{1,3}$ Roberto Verzicco $\odot{ }^{1,4,5}$ Olga Shishkina $\odot,{ }^{2}$ and Detlef Lohse $\oplus^{1,2, *}$ ${ }^{1}$ Physics of Fluids Group, Max Planck Center for Complex Fluid Dynamics, MESA+ Institute and J.M.Burgers Center for Fluid Dynamics, University of Twente, P.O. Box 217, 7500 AE Enschede, Netherlands

${ }^{2}$ Max Planck Institute for Dynamics and Self-Organisation, Am Fassberg 17, 37077 Göttingen, Germany

${ }^{3}$ Department of Modern Mechanics, University of Science and Technology of China, Hefei 230027, China

${ }^{4}$ Dipartimento di Ingegneria Industriale, University of Rome 'Tor Vergata', Via del Politecnico 1, Roma 00133, Italy

${ }^{5}$ Gran Sasso Science Institute-Viale F. Crispi, 7, 67100 L'Aquila, Italy

(Received 29 April 2020; accepted 9 September 2020; published 9 October 2020)

\begin{abstract}
Many natural and industrial turbulent flows are subjected to time-dependent boundary conditions. Despite being ubiquitous, the influence of temporal modulations (with frequency $f$ ) on global transport properties has hardly been studied. Here, we perform numerical simulations of Rayleigh-Bénard convection with time periodic modulation in the temperature boundary condition and report how this modulation can lead to a significant heat flux (Nusselt number $\mathrm{Nu}$ ) enhancement. Using the concept of Stokes thermal boundary layer, we can explain the onset frequency of the $\mathrm{Nu}$ enhancement and the optimal frequency at which $\mathrm{Nu}$ is maximal, and how they depend on the Rayleigh number Ra and Prandtl number Pr. From this, we construct a phase diagram in the 3D parameter space $(f, \mathrm{Ra}, \mathrm{Pr})$ and identify the following: (i) a regime where the modulation is too fast to affect $\mathrm{Nu}$; (ii) a moderate modulation regime, where $\mathrm{Nu}$ increases with decreasing $f$, and (iii) slow modulation regime, where $\mathrm{Nu}$ decreases with further decreasing $f$. Our findings provide a framework to study other types of turbulent flows with timedependent forcing.
\end{abstract}

DOI: 10.1103/PhysRevLett.125.154502

Turbulent flows driven by time-dependent forcing are common in nature and industrial applications $[1,2]$. For example, Earth's atmosphere circulation is driven by periodical heating from solar radiation, the ocean tidal current by periodical gravitational attractions from both the Moon and the Sun, and the blood circulation by the beating heart.

In periodically driven turbulence in shear flows, a meanfield theory has been used to analyze the resonance maxima of the Reynolds number [3,4]. Periodic forcing in other turbulent systems, for example, in the homogeneous isotropic turbulence [5-8], pipe flow [9-12], channel flow $[13,14]$, Taylor-Couette flow $[15,16]$, and Rayleigh-Bénard (RB) convection [17-19], is also shown to have highly nontrivial response properties.

Here we picked turbulent Rayleigh-Bénard convection as a model system to study how time periodic modulation of temperature boundary condition influences global heat transport. The RB system, consisting of a fluid layer heated from below and cooled from above, has been extensively studied as the paradigmatic and well-defined system for convective thermal turbulence [20-22]. Also, several modulation methods have been studied for that system, such as bottom temperature modulation $[17,19,23]$, rotation modulation [18,24], and gravity modulation [25,26]. Intuitively, one may expect that the modulation effect on time-averaged global quantities is limited because the net force averaged over a cycle vanishes. Indeed, with bottom temperature modulation in experiments, only a small enhancement $(\approx 7 \%)$ of the heat flux has been observed so far $[17,19]$. However, in those experiments, the effects of modulation in temperature have not yet been fully explored because of the experimental challenge in having a broad range of modulation frequency due to thermal inertia of the plates. Note that also in numerical simulations thermal inertial can straightforwardly be treated $[27,28]$, but in this study, for conceptional clarity, we keep the problems of thermal transport in the RB cell and in the plates disentangled and assume perfect conductivity of the plates.

In this Letter, we numerically study modulated RB convection within a wide range (more than 4 orders of magnitude) of modulation frequency at the bottom plate temperature and observe a significant $(\approx 25 \%)$ enhancement in heat transport. To explain our findings, we show the relevance of the Stokes thermal boundary layer (BL), which is analogous to the classical one for an oscillating plate [29], in determining the transitional frequency for the heat transport enhancement and the optimal frequency for the maximal heat transport. In particular, we calculate the transition between the different regimes in phase space and show how they depend on the Rayleigh and Prandtl numbers, which represent the ratios between buoyancy and viscosity and between momentum diffusivity and thermal diffusivity, respectively. Our modulation method 
is complementary to hitherto used concepts of using additional body force or modifying the spatial structure of the system to enhance heat transport, for example, adding surface roughness [30-32], shaking the convection cell [33], including additional stabilizing forces through geometrical modification [34-36], rotation [37], inclination [38,39], or a second stabilizing scalar field [40].

Next to the aspect ratio of the horizontal and vertical extensions of the container, the dimensionless control parameters are the Rayleigh number $\mathrm{Ra}=\alpha g H^{3} \Delta /(\nu \kappa)$ and the Prandtl number $\operatorname{Pr}=\nu / \kappa$, with $\alpha, \nu$, and $\kappa$ being, respectively, the thermal expansion coefficient, kinematic viscosity and thermal diffusivity of the fluid, $g$ the gravitational acceleration, and $\Delta$ the temperature difference between the bottom and top boundaries. The time, length, and temperature are made dimensionless by the free-fall time $\tau=\sqrt{H / \alpha g \Delta}$, the height $H$ of the container, and the temperature difference $\Delta$, respectively. In the following, all quantities are dimensionless, if not otherwise explicitly stated. In the periodically modulated RB, we give a sinusoidal modulation signal to the bottom temperature as

$$
\theta_{\mathrm{bot}}=1+A \cos (2 \pi f t) \text {. }
$$

For modulated RB, two more parameters have to be introduced, namely the modulation frequency $f$ and its amplitude $A$, which is kept fixed in this study, $A=1$. The efficiency of the heat transport and flow strength in the system are represented in terms of the Nusselt number $\mathrm{Nu}$ (the dimensionless heat flux) and the Reynolds number $\operatorname{Re}$ (the ratio between inertia and viscous forces). Direct numerical simulation (DNS) for incompressible OberbeckBousinesq flow are employed [41]; the numerical details are provided in the Supplemental Material [42]. The DNS are conducted in a two-dimensional square box with no-slip and impermeable boundary conditions (BCs) for all walls. The explored parameter range spans $10^{7} \leq \mathrm{Ra} \leq 10^{9}$, $1 \leq \operatorname{Pr} \leq 8$, and $10^{-4} \leq f \leq 4$. We are aware of the limitation of the two-dimensionality of the system on which we focus, but in particular for $\operatorname{Pr} \geq 1$ two- and three-dimensional RB convections show very close similarities and features [44]. To support that our results are also relevant for 3D RB, we conduct a set of three-dimensional DNS in a cubic box at $\mathrm{Ra}=10^{8}$ and $\mathrm{Pr}=4.3$ with various frequencies.

Figure 1(a) shows how the global convective heat flux $\mathrm{Nu}$ depends on the modulation frequency $f$ at fixed $\mathrm{Pr}=4.3$ (corresponding to water). The dependence of $\mathrm{Nu}$ on $f$ exhibits a universal trend for both, two- and threedimensional results, which is independent of Ra: When $f$ is large enough, $\mathrm{Nu}$ is not sensitive to the modulation frequency, and the value is close to the value $\mathrm{Nu}_{0}$ for the case without modulation. However, when $f$ is below a certain onset frequency (denoted as $f_{\text {onset }}$ ), there exists an intermediate regime with significantly enhanced heat flux

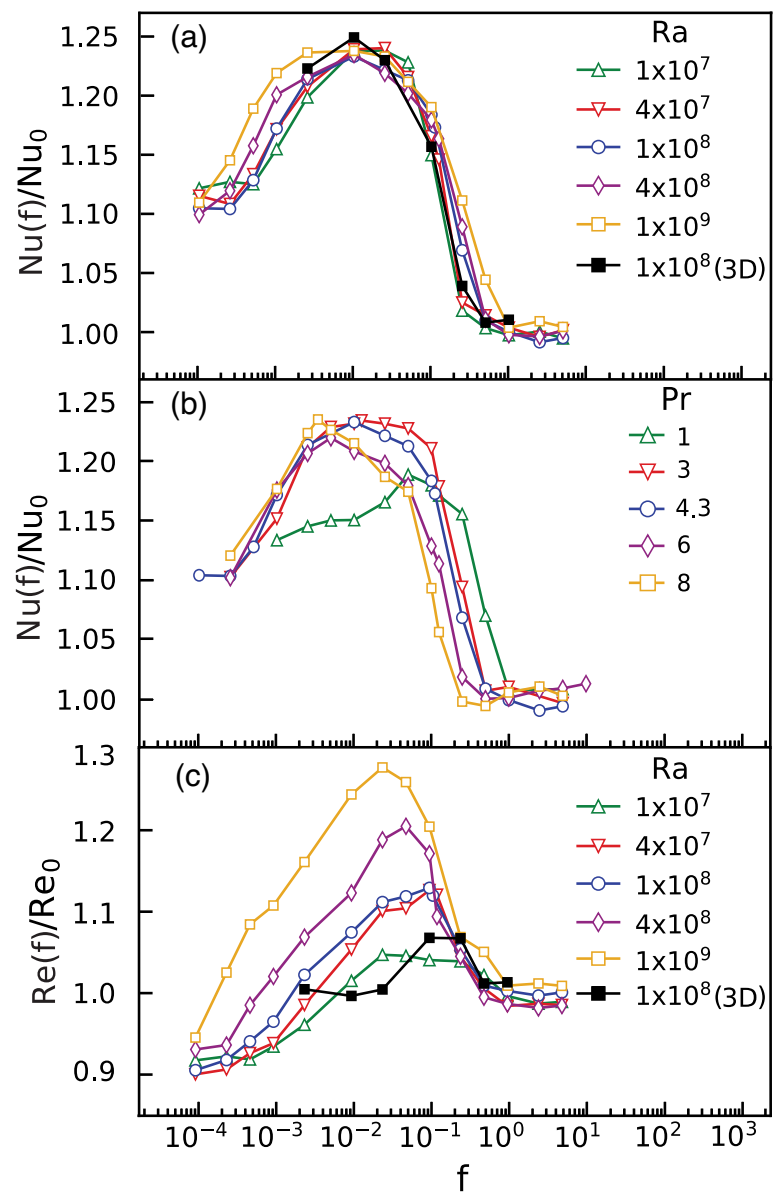

FIG. 1. (a) Modulated frequency dependence of the Nusselt number $\mathrm{Nu}(f)$, normalized by $\mathrm{Nu}_{0}=\mathrm{Nu}(f=0)$, for different Rayleigh numbers and fixed $\mathrm{Pr}=4.3$. (b) $\mathrm{Nu}(f) / \mathrm{Nu}_{0}$ for different Prandtl numbers and fixed $\operatorname{Ra}=10^{8}$. (c) Global $\operatorname{Re}(f)$ normalized by the $\operatorname{Re}_{0}=\operatorname{Re}(f=0)$ for different Rayleigh numbers and fixed $\operatorname{Pr}=4.3$.

as compared to $\mathrm{Nu}_{0}$. With $f$ decreasing further, one observes an optimal frequency $f_{\text {opt }}$ at which Nu is maximal with an enhancement of approximately $25 \%$. Such a large enhancement of $\mathrm{Nu}$ is highly nontrivial because the time-averaged temperature of the bottom plate is still fixed at 1 , and we only have changed the bottom temperature from a steady value to a time periodic signal. In Fig. 1(b), we further examine the $\mathrm{Nu}(f)$ dependence for different $\mathrm{Pr}$, with $\mathrm{Ra}$ fixed at $10^{8}$. One can see that both $f_{\text {onset }}$ and $f_{\text {opt }}$ are much more sensitive to Pr than to Ra.

We first examine whether the transition is related to the strength of the large-scale circulation (LSC). Figure 1(c) shows the global Reynolds number Re as function of $f$ for various $\mathrm{Ra}$, from which we can see that Re is maximized at a Ra-dependent frequency $f_{\text {opt,Re }}$ (see Reynolds resonance in Supplemental Material [42] for further analysis of $f_{\text {opt,Re }}$ ). However, when comparing the $\mathrm{Nu}$ and Re behavior, one observes that the position of the strongest LSC does not correspond to that of the maximum heat transport 

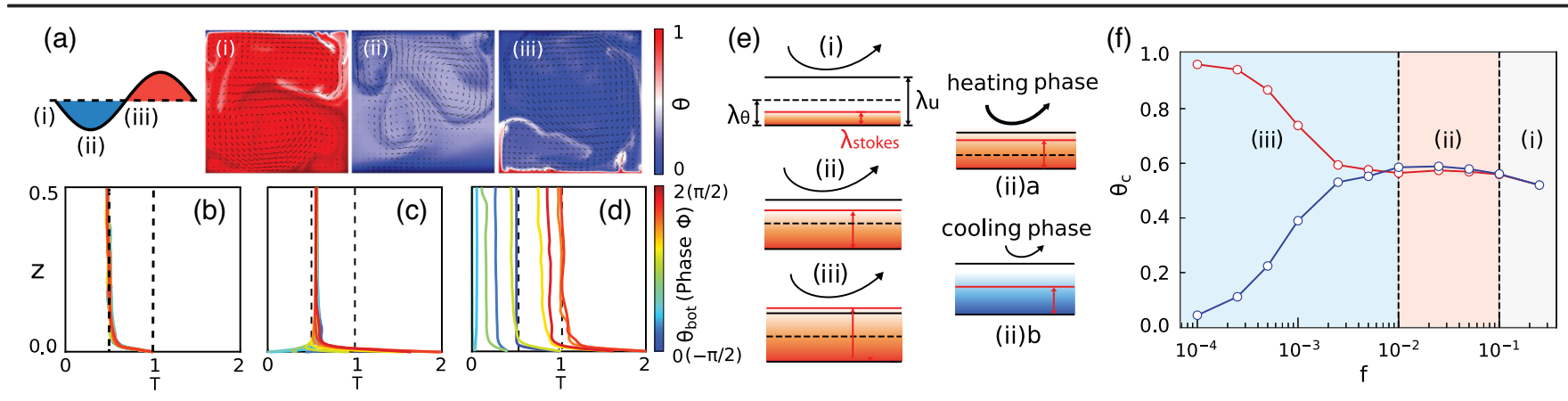

FIG. 2. (a) Instantaneous temperature fields at different phases in one modulation period for $\operatorname{Ra}=10^{8}, \operatorname{Pr}=4.3, f=10^{-3}$. (b)-(d) Phase-averaged temperature profiles during one period for $\operatorname{Ra}=10^{8}, \operatorname{Pr}=4.3$ and different modulation frequencies, namely (b) without modulation; (c) $f=10^{-1}$; (d) $f=10^{-4}$. The horizontal axis is the temperature and the vertical axis is the height. The colorbar shows the bottom temperature (phase angle) from $0(-\pi / 2)$ to $2(\pi / 2)$. (e) Sketch of the relations between the three BLs [Stokes thermal $\mathrm{BL}\left(\lambda_{S}\right)$, thermal BL $\left(\lambda_{\theta}\right)$, momentum BL $\left(\lambda_{u}\right)$ ] for the three regimes $\left(\operatorname{Pr}=4.3\right.$ ): (i) $\lambda_{u}>\lambda_{\theta}>\lambda_{S}$; (ii) $\lambda_{u}>\lambda_{S}>\lambda_{\theta}$; (iii) $\lambda_{S}>\lambda_{u}>\lambda_{\theta}$. Arrows represent the flow in the bulk. (b) Sketch of two different phases during one period for regime ii: (a) heating phase when $\theta_{\text {bot }}>1$ and (b) cooling phase when $\theta_{\text {bot }}<1$. (f) Phase-averaged center temperature for $\operatorname{Ra}=10^{8}, \operatorname{Pr}=4.3$. The red (blue) curve represents the phase when the bottom temperature is maximal (minimal). The dashed lines (from right to left) correspond to $f_{\text {onset }}$ and $f_{\text {opt }}$ for $\mathrm{Nu}$.

$\left(f_{\text {opt }} \neq f_{\text {opt,Re }}\right)$. What physics then governs the transitions between the regimes of heat flux?

To gain insight into this problem, we analyze how the flow structure is changed under modulation. Figure 2(a) shows the temperature fields at different phases of modulation at $f=10^{-3}$. During the heating phase $\left(\theta_{\mathrm{bot}}>1\right)$, frequent plume emissions are observed near the bottom plate. On the contrary, during the cooling phase $\left(\theta_{\text {bot }}<1\right)$, there are no plume emissions from the bottom plate because of the stable stratification near that surface, and the resulting weakening of the circulation.

We further calculate the conditional average of the temperature profiles at different phases, and compare these profiles for different modulation frequencies in Figs. 2(b)-2(d). Without modulation, we recover traditional RB with a mean bulk temperature of 0.5 [Fig. 2(b)]. When $f=10^{-1}$ as shown in Fig. 2(c), the temperature adjacent to the bottom is significantly affected by modulation, whereas the bulk value is still close to 0.5 . However, the overall influence of the modulation is limited because it is too fast to be sensed by the system. With decreasing modulation frequency, the bulk temperature is more and more influenced by the modulation (see Fig. 1 of the Supplemental Material [42]). This suggests that there exists a certain length scale which characterizes how deep the influence of the modulation can penetrate into the convective flow.

To better understand this length scale, we recall the classical Stokes problem. In this flow, a BL is created by an oscillating solid surface with modulating velocity $U \cos (2 \pi f t)$. Likewise, in modulated RB, we can draw the analogy between an oscillating velocity and the oscillating temperature $\theta^{\prime}$, where $\theta^{\prime}=\theta-\bar{\theta}(z)$, with $\bar{\theta}(z)$ being the temporally averaged temperature at height $z$. The governing equation and corresponding BCs are

$$
\begin{aligned}
& \partial \theta^{\prime} / \partial t=(R a P r)^{-1 / 2} \partial^{2} \theta^{\prime} / \partial z^{2}, \\
& \theta^{\prime}(0, t)=A \cos (2 \pi f t), \quad \theta^{\prime}(\infty, t)=0 .
\end{aligned}
$$

The analytical solution of this PDE is an exponential profile:

$$
\theta^{\prime}(z, t)=A e^{-z / \lambda_{S}} \cos \left(2 \pi f t-z / \lambda_{S}\right),
$$

with the so-called Stokes thermal BL thickness

$$
\lambda_{S}=\pi^{-1 / 2} f^{-1 / 2} \operatorname{Ra}^{-1 / 4} \operatorname{Pr}^{-1 / 4},
$$

which is the penetration depth of the disturbance created by the oscillating temperature at the boundary. The distortion [Eq. (3)] travels as a transverse wave through the fluid.

From Eq. (4) one can see that the thickness $\lambda_{S}$ of the Stokes thermal BL decreases with increasing modulation frequency. Depending on the relative thicknesses of $\lambda_{S}$, that of the thermal BL $\lambda_{\theta}$, and that of the momentum BL $\lambda_{u}$, we can obtain three regimes shown in Fig. 2(e). Here we have restricted our discussion to $1 \leq \operatorname{Pr} \leq 8$, where $\lambda_{u} \geq \lambda_{\theta}$.

Regime (i): for $\lambda_{S}<\lambda_{\theta}<\lambda_{u}$, the effect of modulation is confined inside the thermal BL, which is also shown by the temperature profiles in Fig. 2(c). In such case, the effect of modulation is negligible and the heat transport is almost unaffected.

Regime (ii): for $\lambda_{\theta} \leq \lambda_{S}<\lambda_{u}$, the plume emission, which occurs at the edge of the thermal BL, can now be influenced by the modulation [Fig. 2(e)], leading to the enhancement of heat transport. We note that in thermal convection with a rough plate, a $\mathrm{Nu}$ enhancement can also be observed when the thermal BL is perturbed by roughness $[45,46]$. Here, we understand the enhancement in $\mathrm{Nu}$ by the following mechanism: In the heating phase $\left(\theta_{\mathrm{bot}}>1\right)$, there is a 
stronger convective flow and more energetic plumes, as compared to the case without modulation. This can be seen from the value of $\mathrm{Nu}$ at the top plate (see Supplementary Material [42]), where it increases to the values above that without the modulation during the heating phase. However, in the cooling phase, $\mathrm{Nu}$ starts to decline but still remains at values comparable to that without modulation, due to the remaining convective flow. Therefore, there is a net increase in $\mathrm{Nu}$ after one cycle, as compared to the value of $\mathrm{Nu}$ without time-dependent modulation.

Regime (iii): for $\lambda_{\theta}<\lambda_{u} \leq \lambda_{S}$, the effect of temperature modulation penetrates into the bulk region occupied by the LSC. The role of the bulk flow is to efficiently bring the injected hot/cold fluid near the plates during the heating/ cooling phase to the center of the system. Therefore, the center temperature also varies with the phases as seen in Fig. 2(f), in contrast to the situation in regimes (i) and (ii). As a result, at the peak of the heating phase $\left(\theta_{\text {bot }}=2\right)$, the temperature difference between the bottom plate and the bulk cannot be maintained at $\Delta \theta \simeq 1.5$. The thermal driving in the heating phase becomes weaker for smaller $f$, and the global $\mathrm{Nu}$ is expected to decrease for decreasing $f$. When the frequency decreases further and goes to 0 , the limiting value of $\mathrm{Nu}$ should be higher than without modulation. This is because the asymptotic value of $\mathrm{Nu}$ is the integral of the $\mathrm{Nu}(\mathrm{Ra}$ ) (ranging from 0 to $2 \mathrm{Ra}$ ). However, the relation between $\mathrm{Nu}$ and $\mathrm{Ra}$ is nonlinear [47].

According to the physical picture of the three regimes, we compare the relative BL thickness to obtain the boundaries of the regimes, i.e., $f_{\text {onset }}(\operatorname{Ra}, \operatorname{Pr})$ and $f_{\text {opt }}(\operatorname{Ra}, \operatorname{Pr})$. First, we make use of the relations $\lambda_{\theta} \sim \mathrm{Nu}^{-1}$ and $\lambda_{u} \sim \mathrm{Re}^{-1 / 2}$ for the thermal and momentum BL thicknesses. Then we use the Grossmann-Lohse model for the scaling of $\mathrm{Nu}(\mathrm{Ra}, \mathrm{Pr})$ and $\operatorname{Re}(\mathrm{Ra}, \mathrm{Pr})$ in the $\mathrm{I}_{\infty}$ regime (for large $\mathrm{Pr}$ ) [47,48]: $\mathrm{Nu} \sim$ $\operatorname{Pr}^{0} \operatorname{Ra}^{1 / 3}$ and $\operatorname{Re} \sim \operatorname{Pr}^{-1} \operatorname{Ra}^{2 / 3}$. The onset frequency $f_{\text {onset }}$ corresponds to the transition between regime $\mathrm{i}$ and regime ii $\left(\lambda_{S} \sim \lambda_{\theta}\right)$, and we obtain

$$
f_{\text {onset }} \sim \operatorname{Ra}^{1 / 6} \operatorname{Pr}^{-1 / 2} .
$$

The optimal frequency $f_{\text {opt }}$ corresponds to the transition between regime ii and regime iii $\left(\lambda_{S} \sim \lambda_{u}\right)$, and we have

$$
f_{\text {opt }} \sim \operatorname{Ra}^{1 / 6} \operatorname{Pr}^{-3 / 2}
$$

To check these predictions for $f_{\text {onset }}$ and $f_{\text {opt }}$, we replot $\mathrm{Nu}(f)$ for various $\mathrm{Ra}$ but now versus the rescaled frequency $f \mathrm{Ra}^{-1 / 6}$; see Fig. 3(a) ( $\mathrm{Pr}=4.3$ fixed). Indeed, the figure shows rather good collapses around the onset. Next, we vary $\mathrm{Pr}$ for a fixed $\mathrm{Ra}=10^{8}$ and plot $\mathrm{Nu}$ versus the correspondingly rescaled frequencies, namely $f \operatorname{Pr}^{1 / 2}$ for the onset [Fig. 3(b)] and $f \operatorname{Pr}^{3 / 2}$ for the optimum [Fig. 3(c)]. Indeed, one can see the rescaled frequencies (horizontal axis) collapse well, indicating that equations (5) and (6) correctly predict the onset frequency and the optimal frequency for all Pr.

Finally, we present the phase diagram in the $f$ vs Ra and the $f$ vs Pr parameter spaces in Figs. 3(d) and 3(e).
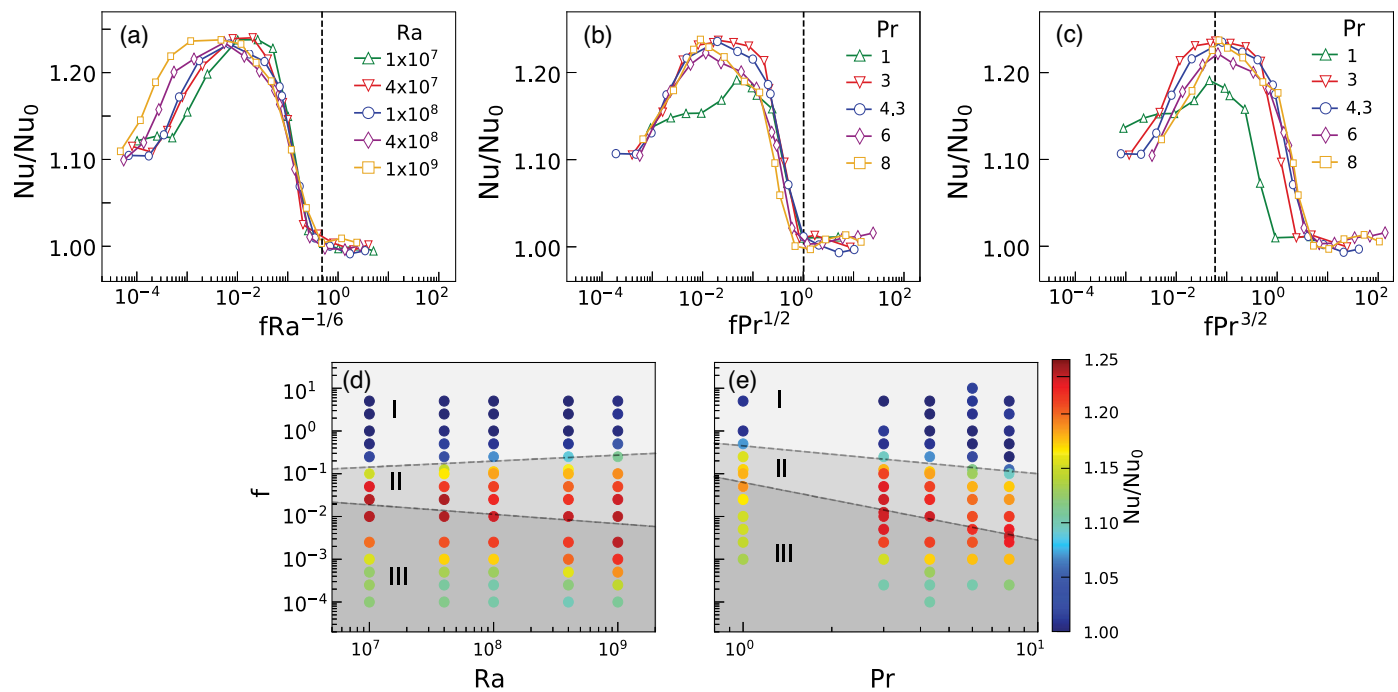

FIG. 3. (a) Normalized $\mathrm{Nu}$ as a function of $f R a^{-1 / 6}$, for different $\operatorname{Ra}$ and $\operatorname{Pr}=4.3$, (b) $f \operatorname{Pr}^{1 / 2}$. (c) $f \operatorname{Pr}^{3 / 2}$ for different $\operatorname{Pr}$ and $\mathrm{Ra}=10^{8}$. Dashed lines show the onset frequency [where $\mathrm{Nu}(f)$ starts to be affected, $\mathrm{Nu}(f) / \mathrm{Nu}_{0}=1.01$ ] or optimal frequency [where $\mathrm{Nu}(f)$ reaches the maximum], averaged for different Ra or Pr. Phase diagram (a) in the $f$ vs Ra and (b) in the $f$ vs Pr parameter spaces. In (a), the lower dashed line shows the optimal frequency $f_{\text {opt }}=0.65 \mathrm{Ra}^{-0.22}$ that corresponds to the maximal Nu. The upper dashed line shows the onset frequency $f_{\text {onset }}=0.015 \mathrm{Ra}^{0.14}$ that corresponds to the onset of the heat flux enhancement. In (b), the lower dashed line shows the optimal frequency $f_{\text {opt }}=0.06 \operatorname{Pr}^{-1.35}$, while the upper one shows the onset frequency $f_{\text {onset }}=0.45 \operatorname{Pr}^{-0.65}$. The prefactors originate from fits to the DNS data for $f_{\text {opt }}$ and $f_{\text {onset }}$ [set to occur when $\mathrm{Nu}(f) / \mathrm{Nu}_{0}=1.01$ ]; see Supplemental Material [42] for details on the fitting. 
We classify three regimes: classical RB regime (i), modulation-enhancement regime (ii), and modulation-reduction regime (iii). The boundary between the regimes is found by fitting the numerically obtained $f_{\text {onset }}$ and $f_{\text {opt }}$. The fitting scaling relations for onset and optimum $\left(f_{\text {onset }} \sim \mathrm{Ra}^{0.14} \operatorname{Pr}^{-0.65}, f_{\text {opt }} \sim \mathrm{Ra}^{-0.22} \operatorname{Pr}^{-1.35}\right)$ show a good agreement with the derived ones $\left(f_{\text {onset }} \sim \mathrm{Ra}^{1 / 6} \mathrm{Pr}^{-1 / 2}\right.$, $f_{\text {opt }} \sim \mathrm{Ra}^{1 / 6} \mathrm{Pr}^{-3 / 2}$ ) except $f_{\text {opt }}$ vs $\mathrm{Ra}$, corresponding to $\lambda_{S} \sim \lambda_{u}$. We notice that in our model, $\lambda_{S}$ is obtained based on a diffusion equation. The neglected advection term can become significant, particularly in regime iii where the Stokes BL may penetrate into the bulk. It, therefore, imposes uncertainty in estimating the weak Ra dependence of $\lambda_{\text {opt }}$. Our explored parameter range only spans $1<\operatorname{Pr}<8$ due to extreme costs to explore a wider range. But our model is general for various $\mathrm{Pr}$, as long as the boundary layers exist and follow the given scaling relations. These obviously no longer hold for extreme Pr values (i.e., very large Pr when the flow becomes laminar and very small Pr, when $\lambda_{u}<\lambda_{\theta}$ ). Moreover, our model indicates the relation of the magnitude of $\mathrm{Nu}$ enhancement with $\mathrm{Ra}$ and Pr. From Figs. 1(a) and 1(b), the maximal $\mathrm{Nu}$ enhancement increases as $\operatorname{Pr}$ increases while it is independent of Ra. This is because $f_{\text {onset }}$ and $f_{\text {opt }}$ have the same scaling with Ra but different scalings with Pr, as shown in Eqs. (5) and (6). As Pr increases, the gap between $f_{\text {onset }}$ and $f_{\text {opt }}$ becomes larger, and Nu keeps increasing in between. Therefore, the maximal $\mathrm{Nu}$ increases with increasing Pr.

In conclusion, our results have substantial implications for the investigation of modulated convection systems. For a wide range of parameters in the three-dimensional parameter space (modulation frequency $f$, Rayleigh number Ra, and Prandtl number Pr), we have demonstrated how the global heat transport efficiency can be enhanced through temperature modulation in both two- and threedimensional simulations. The high similarity between 2D and 3D DNS results supports that our results are applicable in both cases and robust. Based on the heat transfer enhancement, we can identify three different regimes: the classical RB regime for fast modulation, an intermediate regime in which the modulation leads to increasing $\mathrm{Nu}$ enhancement, and the slow modulation regime in which it leads to decreasing $\mathrm{Nu}$ enhancement. The transitions between the regimes are well predicted by the relative thicknesses of thermal, momentum, and Stokes thermal BLs. Our concept of explaining global transport properties in modulated BL flows by the relative thicknesses of the three relevant BLs can also be extended to the angular velocity transfer in modulated turbulent Taylor-Couette flow, or to the kinetic energy transfers in modulated turbulent pipe flow.

This work was supported by the Priority Programme SPP 1881 Turbulent Superstructures of the Deutsche Forschungsgemeinschaft and by NWO via the Zwaartekrachtprogramma MCEC and an ERC-Advanced
Grant under Project No. 740479. This work was partly carried out on the national e-infrastructure of SURFsara. We also gratefully acknowledge support by the Balzan Foundation.

*d.lohse@utwente.nl

These authors contributed equally to this work.

[1] E. Berger and R. Wille, Periodic flow phenomena, Annu. Rev. Fluid Mech. 4, 313 (1972).

[2] S. H. Davis, The stability of time-periodic flows, Annu. Rev. Fluid Mech. 8, 57 (1976).

[3] D. Lohse, Periodically kicked turbulence, Phys. Rev. E 62 , 4946 (2000).

[4] A. von der Heydt, S. Grossmann, and D. Lohse, Response maxima in modulated turbulence, Phys. Rev. E 67, 046308 (2003).

[5] A. von der Heydt, S. Grossmann, and D. Lohse, Response maxima in modulated turbulence. II. Numerical simulations, Phys. Rev. E 68, 066302 (2003).

[6] A. K. Kuczaj, B. J. Geurts, and D. Lohse, Response maxima in time-modulated turbulence: Direct numerical simulations, Eur. Phys. Lett. 73, 851 (2006).

[7] W. J. Bos and R. Rubinstein, Mixing in modulated turbulence. Analytical results, Comput. Fluids 151, 102 (2017).

[8] Y. Yang, R. Chahine, R. Rubinstein, and W. J. Bos, Passive scalar mixing in modulated turbulence, Fluid Dyn. Res. 51, 045501 (2019).

[9] T. Jelly, R. Chin, S. Illingworth, J. Monty, I. Marusic, and A. Ooi, A direct comparison of pulsatile and non-pulsatile rough-wall turbulent pipe flow, J. Fluid Mech. 895, R3 (2020).

[10] Z. Cheng, T. Jelly, S. Illingworth, I. Marusic, and A. Ooi, Forcing frequency effects on turbulence dynamics in pulsatile pipe flow, Int. J. Heat Fluid Flow 82, 108538 (2020).

[11] P. Papadopoulos and A. Vouros, Pulsating turbulent pipe flow in the current dominated regime at high and very-high frequencies, Int. J. Heat Fluid Flow 58, 54 (2016).

[12] S. He and J. Jackson, An experimental study of pulsating turbulent flow in a pipe, Eur. J. Mech. B 28, 309 (2009).

[13] C. Weng, S. Boij, and A. Hanifi, Numerical and theoretical investigation of pulsatile turbulent channel flows, J. Fluid Mech. 792, 98 (2016).

[14] K. Bhaganagar, Direct numerical simulation of unsteady flow in channel with rough walls, Phys. Fluids 20, 101508 (2008).

[15] R. A. Verschoof, A. K. Te Nijenhuis, S. G. Huisman, C. Sun, and D. Lohse, Periodically driven Taylor-Couette turbulence, J. Fluid Mech. 846, 834 (2018).

[16] C. Barenghi and C. Jones, Modulated Taylor-Couette flow, J. Fluid Mech. 208, 127 (1989).

[17] X. L. Jin and K. Q. Xia, An experimental study of kicked thermal turbulence, J. Fluid Mech. 606, 133 (2008).

[18] S. Sterl, H.-M. Li, and J.-Q. Zhong, Dynamical and statistical phenomena of circulation and heat transfer in periodically forced rotating turbulent Rayleigh-Bénard convection, Phys. Rev. Fluids 1, 084401 (2016). 
[19] J. J. Niemela and K. R. Sreenivasan, Formation of the "Superconducting" Core in Turbulent Thermal Convection, Phys. Rev. Lett. 100, 184502 (2008).

[20] G. Ahlers, S. Grossmann, and D. Lohse, Heat transfer and large scale dynamics in turbulent Rayleigh-Bénard convection, Rev. Mod. Phys. 81, 503 (2009).

[21] D. Lohse and K. Q. Xia, Small-scale properties of turbulent Rayleigh-Bénard convection, Annu. Rev. Fluid Mech. 42, 335 (2010).

[22] F. Chillà and J. Schumacher, New perspectives in turbulent Rayleigh-Bénard convection, Eur. Phys. J. E 35, 58 (2012).

[23] G. Venezian, Effect of modulation on the onset of thermal convection, J. Fluid Mech. 35, 243 (1969).

[24] B. J. Geurts and R. P. Kunnen, Intensified heat transfer in modulated rotating Rayleigh-Bénard convection, Int. J. Heat Fluid Flow 49, 62 (2014).

[25] J. L. Rogers, M. F. Schatz, J. L. Bougie, and J. B. Swift, Rayleigh-Bénard Convection in a Vertically Oscillated Fluid Layer, Phys. Rev. Lett. 84, 87 (2000).

[26] P. Gresho and R. Sani, The effects of gravity modulation on the stability of a heated fluid layer, J. Fluid Mech. 40, 783 (1970).

[27] R. J. A. M. Stevens, D. Lohse, and R. Verzicco, Sidewall effects in Rayleigh-Bénard convection, J. Fluid Mech. 741, 1 (2014).

[28] Z.-H. Wan, P. Wei, R. Verzicco, D. Lohse, G. Ahlers, and R. J. Stevens, Effect of sidewall on heat transfer and flow structure in Rayleigh-Bénard convection, J. Fluid Mech. 881, 218 (2019).

[29] P. Kundu, I. Cohen, and D. Dowling, Fluid Mechanics (Academic Press, Elsevier, 2001).

[30] H. Jiang, X. Zhu, V. Mathai, R. Verzicco, D. Lohse, and C. Sun, Controlling Heat Transport and Flow Structures in Thermal Turbulence Using Ratchet Surfaces, Phys. Rev. Lett. 120, 044501 (2018).

[31] S. Ciliberto and C. Laroche, Random Roughness of Boundary Increases the Turbulent Convection Scaling Exponent, Phys. Rev. Lett. 82, 3998 (1999).

[32] M. S. Emran and O. Shishkina, Natural convection in cylindrical containers with isothermal ring-shaped obstacles, J. Fluid Mech. 882, A3 (2020).

[33] B.-F. Wang, Q. Zhou, and C. Sun, Vibration-induced boundary-layer destabilization achieves massive heattransport enhancement, Sci. Adv. 6, eaaz8239 (2020).

[34] S.-D. Huang, M. Kaczorowski, R. Ni, and K.-Q. Xia, Confinement-induced Heat-transport Enhancement in Turbulent Thermal Convection, Phys. Rev. Lett. 111, 104501 (2013).

[35] K. L. Chong, S.-D. Huang, M. Kaczorowski, and K.-Q. Xia, Condensation of Coherent Structures in Turbulent Flows, Phys. Rev. Lett. 115, 264503 (2015).
[36] K. L. Chong, Y. Yang, S.-D. Huang, J.-Q. Zhong, R. J. Stevens, R. Verzicco, D. Lohse, and K.-Q. Xia, Confined Rayleigh-Bénard, Rotating Rayleigh-Bénard, and Double Diffusive Convection: A Unifying View on Turbulent Transport Enhancement through Coherent Structure Manipulation, Phys. Rev. Lett. 119, 064501 (2017).

[37] R. J. Stevens, J. Q. Zhong, H. J. Clercx, G. Ahlers, and D. Lohse, Transitions between Turbulent States in Rotating Rayleigh-Bénard Convection, Phys. Rev. Lett. 103, 024503 (2009).

[38] L. Zwirner, R. Khalilov, I. Kolesnichenko, A. Mamykin, S. Mandrykin, A. Pavlinov, A. Shestakov, A. Teimurazov, P. Frick, and O. Shishkina, The influence of the cell inclination on the heat transport and large-scale circulation in liquid metal convection, J. Fluid Mech. 884, A18 (2020).

[39] Q. Wang, Z.-H. Wan, R. Yan, and D.-J. Sun, Multiple states and heat transfer in two-dimensional tilted convection with large aspect ratios, Phys. Rev. Fluids 3, 113503 (2018).

[40] Y. Yang, R. Verzicco, and D. Lohse, From convection rolls to finger convection in double-diffusive turbulence, Proc. Natl. Acad. Sci. U.S.A. 113, 69 (2016).

[41] R. Verzicco and P. Orlandi, A finite-difference scheme for three-dimensional incompressible flows in cylindrical coordinates, J. Comput. Phys. 123, 402 (1996).

[42] See Supplemental Material at http://link.aps.org/ supplemental/10.1103/PhysRevLett.125.154502, which includes Refs. [4,5,40,42], for details of simulations, the data of bulk temperature, phase delay, response amplitude, Reynolds number and Nusselt number time series.

[43] O. Shishkina, R. J. A. M. Stevens, S. Grossmann, R. Verzicco, and D. Lohse, Boundary layer structure in turbulent thermal convection and its consequences for the required numerical resolution, New J. Phys. 12, 075022 (2010).

[44] E. P. van der Poel, R. J. A. M. Stevens, and D. Lohse, Comparison between two- and three-dimensional Rayleigh-Bénard convection, J. Fluid Mech. 736, 177 (2013).

[45] Y.-C. Xie and K.-Q. Xia, Turbulent thermal convection over rough plates with varying roughness geometries, J. Fluid Mech. 825, 573 (2017).

[46] Y.-Z. Zhang, C. Sun, Y. Bao, and Q. Zhou, How surface roughness reduces heat transport for small roughness heights in turbulent Rayleigh-Bénard convection, J. Fluid Mech. 836, R2 (2018).

[47] S. Grossmann and D. Lohse, Scaling in thermal convection: A unifying theory, J. Fluid Mech. 407, 27 (2000).

[48] O. Shishkina, M. S. Emran, S. Grossmann, and D. Lohse, Scaling relations in large-Prandtl-number natural thermal convection, Phys. Rev. Fluids 2, 103502 (2017). 